symptom duration of 6 (range 4-10) days were treated. Mean oxygen saturation was $86 \%$ (range $76-95 \%$ ) before initiating therapy. Mean ferritin was $1297 \mu \mathrm{g} / \mathrm{l}$ (range 347 - 2734), mean IL-6 112 ng/L (range 2.2 - 607.4) and CRP $82.4 \mathrm{mg} / \mathrm{L}$ (range $36.4-125$ ). In all patients we were able to prevent them from intubation and mechanic ventilation, none of our patients died. Fife patients did not need to be referred to the intensive care unit at all, while 9 patients received noninvasive ventilation and high-flow nasal oxygen support. All patients showed typical imaging features of COVID-19 pneumonia at baseline (BL) according to the Radiological Society of North America (RSNA) chest CT classification system. The mean of the global chest CT severity score at BL was 13 (range 7-20) and decreased to 6 (range $0-16$ ) within 1 month which corresponded to a mean reduction of $58 \%$. Chronic fibrotic pulmonary changes were not seen in any patient at BL and after 1 month mild changes were observed in 6 patients. One patient experienced lower abdominal pain, urinary tract infection, gastrointestinal bleeding due to antrum ulcers Forrest III, in another patient atrial fibrillation, urinary tract infection and apoplexy were observed.

Conclusion: In our case series, all patients treated with the combination tocilizumab and anakinra recovered fast and sustained without major infectious side effects, indicating that early interruption of cytocine release might be very effective and safe in preventing patients from mechanical ventilation, death and longterm damage.

Image 1: Chest CT of a patient with active COVID-19 infection before and one month after treatment with a combination of tocilizumab and anakinra

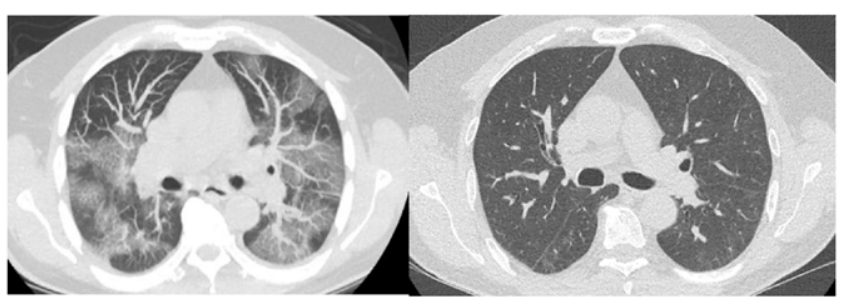

Disclosure of Interests: Hildrun Haibel Speakers bureau: Abbive, Novartis, Janssen, Pfizer, Roche, Consultant of: Janssen, Novartis, Roche, Janis Lucas Vahldiek: None declared, Stefan Angermair: None declared, Michael Schumann: None declared, Britta Siegmund Speakers bureau: Abbvie, CED Service GmbH, Falk, Ferring, Janssen, Novartis and Takeda as a representative of Charité Universitätsmedizin Berlin, Consultant of: Abbvie, Arena, BMS, Boehringer, Celgene, Falk, Janssen, Lilly, Pfizer, Prometheus, Takeda representative of Charité - Universitätsmedizin Berlin, Grant/research support from: Pfizer, Denis Poddubnyy Speakers bureau: AbbVie, Bristol-Myers Squibb, Lilly, MSD, Novartis, Pfizer, and UCB, Consultant of: AbbVie, Biocad, Gilead, GlaxoSmithKline, Eli Lilly, MSD, Novartis, Pfizer, Samsung Bioepis, and UCB, Grant/research support from: AbbVie, Eli Lilly, MSD, Novartis, and Pfizer, Thomas Schneider: None declared. DOI: 10.1136/annrheumdis-2021-eular.2289

\section{POS1210 19 PREVALENCE OF COVID-19 AMONG PATIENTS WITH RHEUMATIC DISEASES: AN OBSERVATIONAL SURVEY DURING THE TWO WAVES IN ITALY}

S. Fasano ${ }^{1}$, I. Pantano ${ }^{1}$, D. Mauro ${ }^{1}$, D. Capocotta ${ }^{2}$, D. lacono ${ }^{1}$, E. Gaggiano ${ }^{1}$, M. D. Pasquale ${ }^{1}$, G. Rozza ${ }^{1}$, C. DI Vico ${ }^{1}$, A. Ruggiero ${ }^{1}$, E. Tirri ${ }^{2}$, F. Ciccia ${ }^{1}$ ${ }^{1}$ University of Campania L. Vanvitelli, Rheumatology Unit, Department of Precision Medicine, Naples, Italy; ${ }^{2}$ Ospedale S. Giovanni Bosco, U.O.S.D. di Reumatologia, Naples, Italy

Background: The new coronavirus 2 (SARS-CoV-2) disease 2019 (COVID-19) is a source of concern for the management of patients suffering from rheumatic and musculoskeletal diseases (RMDs) treated with immunomodulatory therapies (1)

Objectives: We aimed to analyze the prevalence of SARS-CoV-2 infection in patients with RMDs living in Italy.

Methods: During the first wave (March-May 2020) and during the second wave (October-December 2020) of COVID-19, we conducted a survey to investigate the incidence of SARS-CoV-2 infection in patients with RMDs followed at the Rheumatology Unit of the University of Campania, Italy. The demographic data, medication use, the frequency of respiratory symptoms and the incidence of COVID-19 confirmed by nasopharyngeal swab were collected with questionnaires administered by phone. The prevalence of COVID-19 of our cohort was compared to that of the general population (2).

Results: During the first wave, we collected data from 900 patients with RMDs (Table 1): 320 patients with rheumatoid arthritis (RA), 295 patients with spondyloarthropathies (SpA), 283 patients with systemic lupus erythematosus (SLE), 2 patients with vasculitis. $546(60 \%)$ were treated with bDMARD/tsDMARDs. Overall, a total of $11 / 900(1 \%)$ cases were tested for COVID-19 due to compatible symptoms. $2(0.2 \%)$ adult patients treated with bDMARDs were registered as swab test positive by PCR for COVID-19. 2 patients without confirmed COVID-19 developed pneumonia that required admission to hospital. No deaths occurred among the patients with confirmed COVID-19.

During the second wave, data were collected from 470 patients who accepted to take part of the study (Table 1). 49 presented with symptoms that were compatible with COVID-19. 139 patients were tested whereas 30 patients $(6 \%)$ had a swab confirmation of SARS-CoV-2 infection. Among them, $16(53 \%)$ were treated with bDMARDs and a patient was treated with tofacitinib. we found no increase in COVID-19 prevalence in patients treated with bDMARD/tsDMARDs $(p>0.05)$ A patient with SLE developed pneumonia that required admission to hospital and died.

Lacking distinct prevalence data between first and second waves, we found no differences in total COVID-19 prevalence between general population living in Campania $(215.752 / 5.802 .000 ; 3.7 \%)$ and patients with RMDs $(32 / 900 ; 3.5 \%)$ However, we had a significant increase in COVID-19 prevalence in our cohor during the second wave compared to the first. Nevertheless, no increase in mortality or hospitalization was recorded, confirming the safety of immunomodulatory therapies in patients with RMDs.

Conclusion: In this cohort of patients with RMDs in a geographical region with a high prevalence of COVID-19, the risk of SARS-CoV-2 infection does not appear different from that observed in the general population.

\section{REFERENCES:}

[1] Wang L., Wang Y., Ye D. Int J Antimicrob Agents. 2020:105948.

[2] http://www.protezionecivile.gov.it/ (accessed 28.01.21)

Table 1. Demographics and clinical characteristics of 900 patients with rheumatic diseases during the COVID-19 Pandemic.

\begin{tabular}{lcc}
\hline & First wave & Second wave \\
\hline Women, $n$ & $660(73 \%)$ & $366(77 \%)$ \\
Age, years, median (range) & $56(54-57)$ & $53(51-55)$ \\
Rheumatoid Arthritis & $320(35.5 \%)$ & $143(30 \%)$ \\
Spondyloarthritis & $295(32 \%)$ & $110(23 \%)$ \\
Systemic Lupus Erythematosus & $283(31 \%)$ & $217(46 \%)$ \\
Vasculitis & $2(0.2 \%)$ & $1(0.2 \%)$ \\
Prior ILD & $56(6 \%)$ & $22(4.6 \%)$ \\
Smokers & $220(24 \%)$ & $118(25 \%)$ \\
Hydroxychloroquine & $215(23 \%)$ & $155(32 \%)$ \\
Steroids & $337(37 \%)$ & $194(41 \%)$ \\
Prednisone equivalent dose, median (range) & $5(0-75)$ & $5(0-50)$ \\
bDMARD/tsDMARDs & $546(60 \%)$ & $247(52 \%)$ \\
csDMARDS & $387(43 \%)$ & $185(39 \%)$ \\
Angiotensin-converting enzyme (ACE) inhibitors & $178(19.8 \%)$ & $101(21 \%)$ \\
Angiotensin II receptor blockers(ARBs) & $153(17 \%)$ & $61(13 \%)$ \\
Fever & $64(7 \%)$ & $30(6.3 \%)$ \\
Cough & $83(9 \%)$ & $36(7 \%)$ \\
Shortness of breath & $34(3 \%)$ & $15(3 \%)$ \\
Sore throat & $32(3 \%)$ & $11(2.3 \%)$ \\
Rhinorrhoea & $36(3 \%)$ & $11(2.3 \%)$ \\
Headache & $5(0.5 \%)$ & $2(0.4 \%)$ \\
Anosmia & $10(1 \%)$ & $24(5 \%)$ \\
Myalgia & $2(0.2 \%)$ & $1(0.2 \%)$ \\
Gastrointestinal symptoms & $24(2.6 \%)$ & $3(0.6 \%)$ \\
Pneumonia & $2(0.2 \%)$ & $2(0.4 \%)$ \\
Admission to hospital & $2(0.2 \%)$ & $9(1.9 \%)$ \\
Swab confirmation of SARS-CoV-2 infection & 2 & 30 \\
& &
\end{tabular}

Disclosure of Interests: None declared.

DOI: 10.1136/annrheumdis-2021-eular.2297

\section{POS1211 \\ IDENTIFICATION OF COMMON FUNCTIONAL PATHWAYS IN PATIENTS WITH LUPUS AND COVID-19 BY TIME-SERIES ANALYSIS}

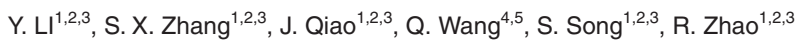
Y. Zhang ${ }^{1,2,3}$, T. Cheng ${ }^{1,2,3}$, M. J. Chang ${ }^{1,2,3}$, G. Y. Liu ${ }^{1}$, J. Luo ${ }^{1}$, P. F. He ${ }^{5}$, X. LI ${ }^{1,2,3} \cdot{ }^{1}$ The Second Hospital of Shanxi Medical University, Department of Rheumatology, Taiyuan, China; ${ }^{2}$ Shanxi Li Xiaofeng Medical Groups, Department of Rheumatology, Taiyuan, China; ${ }^{3}$ Ministry of Education, Key laboratory of Cellular Physiology at Shanxi Medical University, Taiyuan, China;

${ }^{4}$ Shanxi Medical University, Basic Medical College, Taiyuan, China; ${ }^{5}$ Shanxi Medical University, Medical Data Sciences, Taiyuan, China

Background: Systemic lupus erythematosus (SLE) is a chronic autoimmune disorder characterized by abnormal activity of the immune system, producing the autoantibodies directed against nuclear and cytoplasmic antigen ${ }^{1}{ }^{1}$. Infection is known as one of the common trigger factors for SLE. Coronavirus disease in 2019 (COVID-19), a severe acute respiratory syndrome, is now spreading rapidly throughout the world ${ }^{2}$.Though previous studies have addressed the susceptibility of lupus patients to the virus but how patients with SLE deal with COVID-19 is unclear up until now. 